\title{
Situación de las legumbres en Chile: Una mirada agronómica
}

\author{
The status of legumes in Chile: an agronomic view
}

RESUMEN

Las legumbres juegan un rol fundamental en la agricultura moderna; su inclusión en los sistemas agrícolas permite hacer un uso más sostenible de los recursos, ya que mejoran la fertilidad de los suelos, reducen la dependencia a los fertilizantes químicos al fijar el nitrógeno del aire, favorecen la rotación de cultivos tradicionales, principalmente cereales, y mejoran el tenor proteico de los cultivos que le suceden en la rotación. En este artículo se entregan aspectos relacionados con la situación agrícola actual de las legumbres en Chile, con datos estadísticos en cuanto a la evolución de la superficie sembrada, rendimiento, importaciones y requerimiento interno. A su vez, se dan a conocer las principales ventajas agronómicas de este grupo de cultivos y los problemas de manejo más importantes que han Ilevado, a este rubro, a tener la situación actual de desmedro en términos de producción en el país. Finalmente, se presentan una serie de desafíos factibles de llevar a cabo y que podrían revitalizar a este grupo de cultivos tan importantes desde un punto de vista ambiental como alimenticio.

Palabras clave: Legumbres; Fijación de Nitrógeno; Sostenibilidad agrícola; Salud del sistema agrícola; Rotación de cultivos.

\section{ABSTRACT}

Legumes play a fundamental role in modern agriculture; their inclusion in the farming system allows a sustainable use of resources. This is due to the fact that legumes improve soil fertility by fixing atmospheric nitrogen, thus reducing the need for chemical fertilizer. Likewise, they improve crop rotation, mainly in cereal crops, increasing the protein content of successive crops. In this article, we describe aspects of the current legume situation in Chile with statistical data relative to the trends of sowing area, yield, imports and internal demand. This article also presents the agronomical advantages of using legumes and the main problems associated with crop management that has deteriorated production in this country. Finally, we present possible solutions to
Cecilia Baginsky G, Luz Ramos C.

Facultad de Ciencias Agronómicas. Universidad de Chile,

Dirigir correspondencia a: Dra. Cecilia Baginsky. Departamento de Producción Agrícola, Facultad de Ciencias Agronómicas. Universidad de Chile, Av. Santa Rosa 11315, La Pintana, Santiago, Chile. E-mail: cbaginsk@uchile.cl

Este trabajo fue recibido el 15 de junio de 2017. Aceptado para ser publicado: 15 de diciembre de 2017

reinvigorate production of this crop, which is important from both an environmental and nutrition perspective.

Key words: Legumes; Nitrogen Fixation, sustainable agricultural, health of the agricultural system, crop rotation.

\section{INTRODUCCIÓN}

Las leguminosas son cultivos importantes para la salud humana y animal dado su alto aporte proteico, contenido de aminoácidos esenciales, fibra y minerales. En agricultura, contribuyen al aumento del nitrógeno $(\mathrm{N})$ en el suelo, 
por lo que su inclusión en rotaciones de cultivos permite incrementar los rendimientos y disminuir los costos del cultivo sucesor ${ }^{1-3}$. Al mismo tiempo, controlan o reducen la incidencia de enfermedades y malezas y actúan como mejoradoras de la estructura del suelo, por el aporte de materia orgánica tanto en superficie -a través de la incorporación de rastrojos-, como en profundidad -por el crecimiento de sus raíces pivotantes-a lo largo del perfil de suelo. Hay que destacar el bajo consumo de agua asociado a la producción de legumbres, observándose que la huella hídrica es menor que la requerida para la producción de otros alimentos de valor nutricional similar y muy por debajo del de la carne ${ }^{4}$. Este aspecto es relevante, frente al cambio climático existente en la actualidad.

A pesar de los evidentes beneficios, la producción nacional ha disminuido considerablemente en los últimos 30 años. Este hecho está asociado al incremento en los costosde producción, que desfavorece los márgenes de ganancia,y al desincentivo a la siembra, debido al aumento de lasimportaciones producto del bajo precio de las legumbres enel extranjero, las que a su vez son producidas a una mayorescala que en Chile y/o subsidiada. Sumado a ello, los rendimientos se han mantenido constantes en los últimos años producto del bajo nivel tecnológico y al escaso mejoramiento genético de los cultivares, lo que ha provocado que este aumento en los costos no pueda ser compensado por una mayor producción.

El escenario, por tanto, a nivel de la pequeña agricultura que es donde está inserto gran parte del sector productivo de este rubro, no es muy auspicioso. Sin embargo, por las ventajas antes descritas y con el afán de rescatar cultivos como el poroto, que están estrechamente ligados a la identidad nacional, se requiere un input de innovación tanto tecnológica como gastronómica a este grupo de cultivos tan alimenticios y tan necesarios desde un punto de vista agronómico.

\section{Situación productiva de las legumbres}

El término legumbre está asociado a plantas que pertenecen a la familia de las leguminosas y cuyo destino es la producción de granos secos para la alimentación humana y/o animal. Se les ha dado ese nombre para diferenciarlas de otras leguminosas cuyo destino de producción es consumo hortícola (poroto verde, porotos granados, habas frescas), obtención de aceite (soja, maní) o forraje (trébol, alfalfa). En el país, las legumbres más cultivadas son: poroto, garbanzo, lenteja y lupino; sin embargo, también se produce arveja y algo de chicharos 5 .

En Chile, las legumbres son cultivos establecidos en superficies pequeñas, arraigados al quehacer de la agricultura familiar campesina (AFC), que concentran cerca del $80 \%$ de la producción. Es así como a nivel nacional durante la temporada 2015-2016 la superficie alcanzó un total de 27.380 ha (Tabla 1), que corresponden al $3,73 \%$ de la superficie destinada a cultivos anuales (73.4167 ha $)^{6}$. La mayor superficie está destinada al cultivo de lupino con 13.255 ha y cuyo objetivo principal es alimentación animal -entre ellas la de los salmónidos- y exportación para snack. En el caso de los porotos, lentejas, garbanzos, arvejas y chícharos, su principal uso es el consumo interno. La superficie de estas últimas alcanzó las 14.125 ha $(1,92 \%$ de la superficie nacional) y dentro de ellas, la de porotos es la de mayor importancia $(40,81 \%$ de la superficie destinada al cultivo de legumbres) ${ }^{6}$, siendo este cultivo utilizado principalmente para consumo humano. Asimismo, dentro del grupo de las legumbres, el poroto alcanza los mayores rendimientos con 16,1 qqm ha- 1 versus los 11,4 de arveja; 8,6 qqm ha-1 de lenteja y garbanzo y 4,1 de chícharo. La zona de producción de legumbresen Chile se ubica entre la VII y X Región7, siendo la VIII Región la que concentra la mayor superficie $y$, por ende, el mayor volumen de producción (Figura 1).

Tabla 1

Superficie sembrada con legumbres a nivel nacional durante la temporada 2015/16

\begin{tabular}{|lcc|}
\hline Cultivo & Superficie (ha) & $\begin{array}{c}\text { \% Respecto del total } \\
\text { destinado a legumbres }\end{array}$ \\
\hline Arveja & 1.281 & 4,68 \\
Chícharo & 337 & 1,23 \\
Garbanzo & 409 & 1,49 \\
Lenteja & 924 & 3,37 \\
Poroto & 11.174 & 40,8 \\
Lupino & 13.255 & 48,41 \\
& & $\mathbf{2 7 . 3 8 0}$ \\
Total Legumbres & & $\mathbf{1 0 0}$ \\
(Elaboración propia a partir de datos de ODEPA & & \\
\hline
\end{tabular}




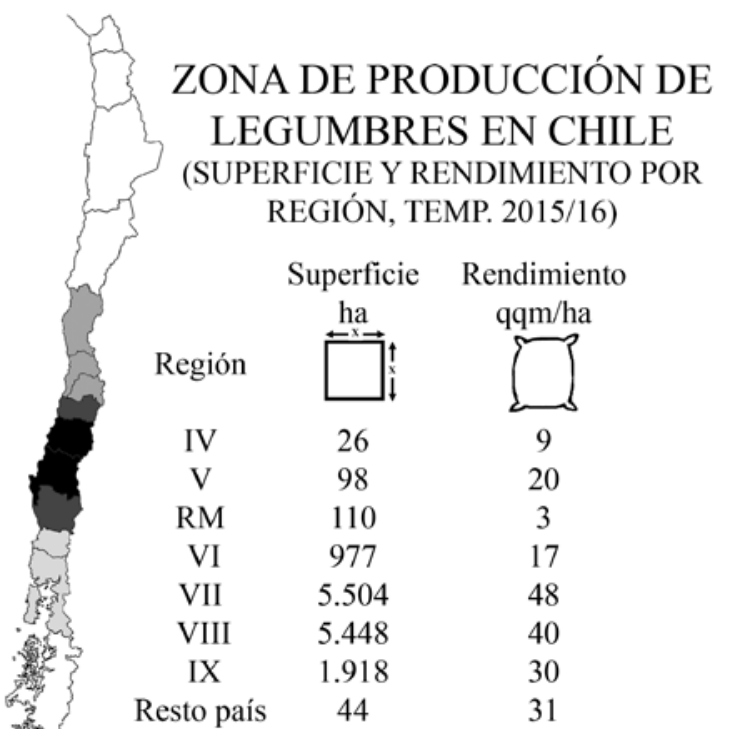

Figura 1. Zonas de producción y rendimientos de legumbres en Chile, temporada 2015/16. (Elaboración propia a partir de datos de ODEPA (7))

Centrando el análisis en las tres legumbres de mayor importancia para consumo humano en el país (poroto, lenteja y garbanzo), se evidencia que en los últimos 30 años la superficie ha decaído de forma considerable (Figura 2, línea punteada) ${ }^{6}$. Por consiguiente, la producción (Figura 2, línea sólida) se ve igualmente disminuida, producto de la baja en la superficie sembrada y a que los rendimientos han sido inestables en el tiempo (Figura 2, línea cortada), y no son capaces de compensar la fuerte baja en la superficie.

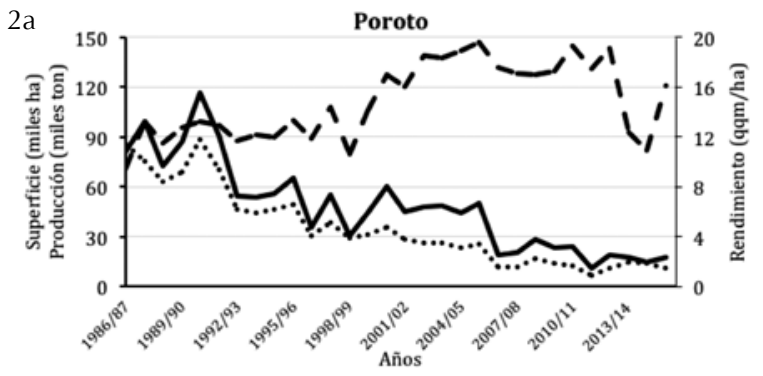

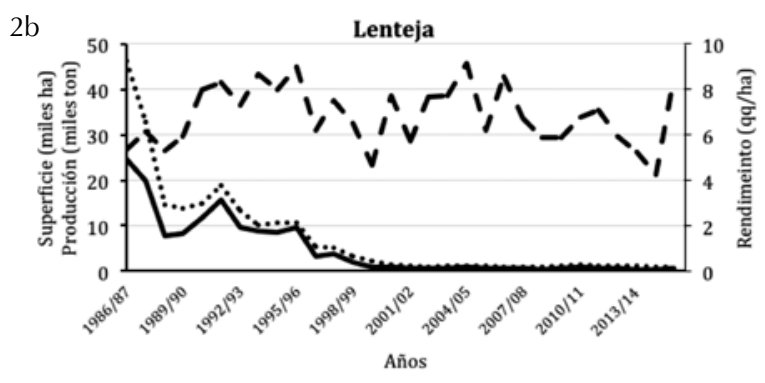
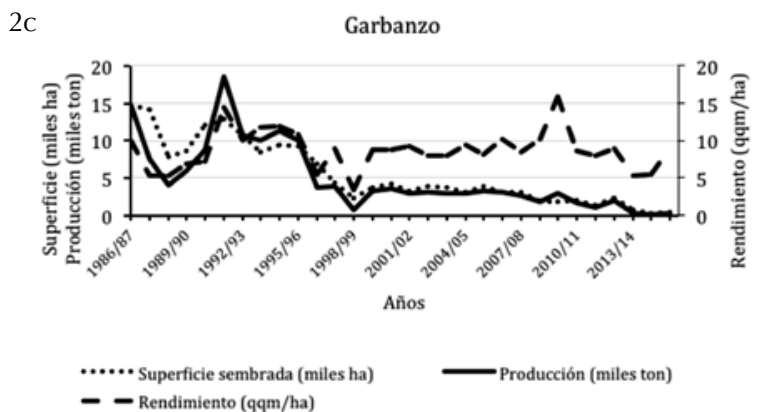

Figura 2. Superficie sembrada, producción y rendimiento de las principales legumbres consumidas a nivel nacional (Elaboración propia a partir de datos de ODEPA ${ }^{6}$.

Las tendencias observadas obedecen a una serie de factores, siendo, los de índole económico, los de mayor importancia dada la escasa rentabilidad de este rubro producto de los bajos rendimientos, alza en los costos de producción y bajo precio de venta. Este último aspecto está asociado al menor precio de las legumbres en el exterior, generado por sistemas agrícolas subsidiados o que producen en grandes extensiones y con economías de escala superiores a las chilenas, donde no prevalece la pequeña agricultura como en Chile. Este hecho ha generado que gran parte de los requerimientos internos sean suplidos por las importaciones (Figura 3), que entran a Chile con precios muy inferiores a los nacionales. Cabe destacar que las importaciones de legumbres, provienen desde países como Canadá (lenteja), China (poroto y garbanzo) o Argentina (Garbanzo) principalmente (Figura 4). Junto a este escenario, se observa una disminución sostenida de la demanda interna de estas legumbres, producto de la baja en el consumo por parte de la población $(1,46$ $\mathrm{kg}$ de poroto, $0,86 \mathrm{~kg}$ de lenteja y $0,25 \mathrm{~kg}$ de garbanzo per cápita al año al 2015), principalmente el de poroto (Figura 5). Este hecho está, en parte asociado, a cambios en el estilo de vida, acceso a otro tipo de proteínas o aspectos socioculturales ${ }^{8}$. 


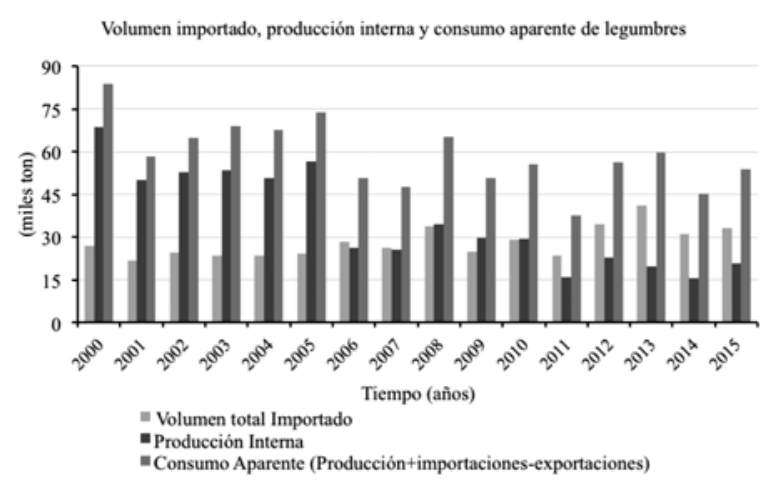

Figura 3. Volumen importado, Producción interna y consumo aparente de legumbres (total país, últimos 16 años). (Elaboración propia a partir de datos de ODEPA (5))

$4 \mathrm{a}$

Porotos

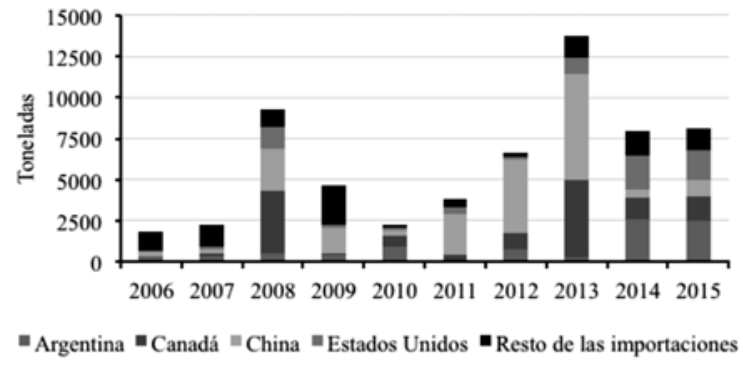

$4 \mathrm{~b}$

Lentejas

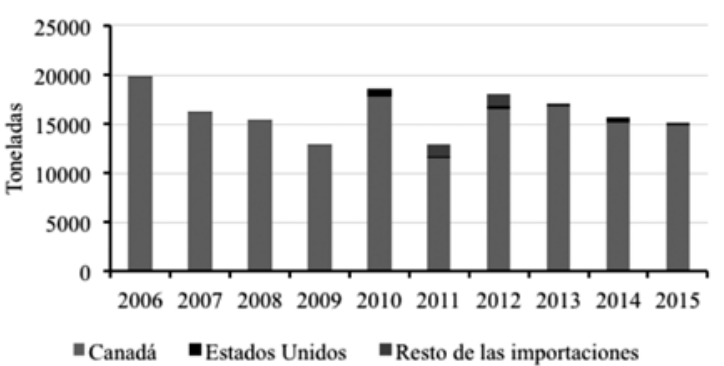

$4 \mathrm{C}$

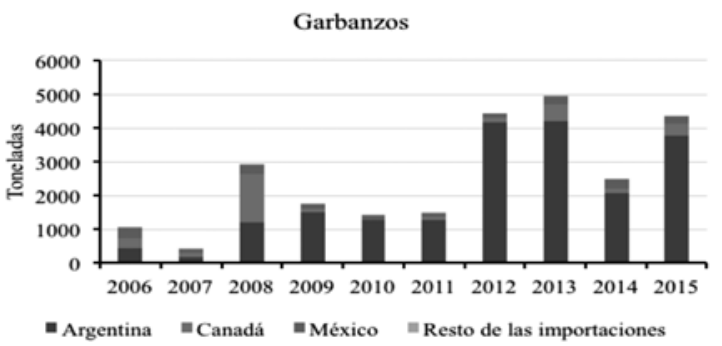

Figura 4. País de origen de las importaciones de las principales legumbres consumidas en Chile. (Elaboración propia a partir de datos de ODEPA ${ }^{5}$ )
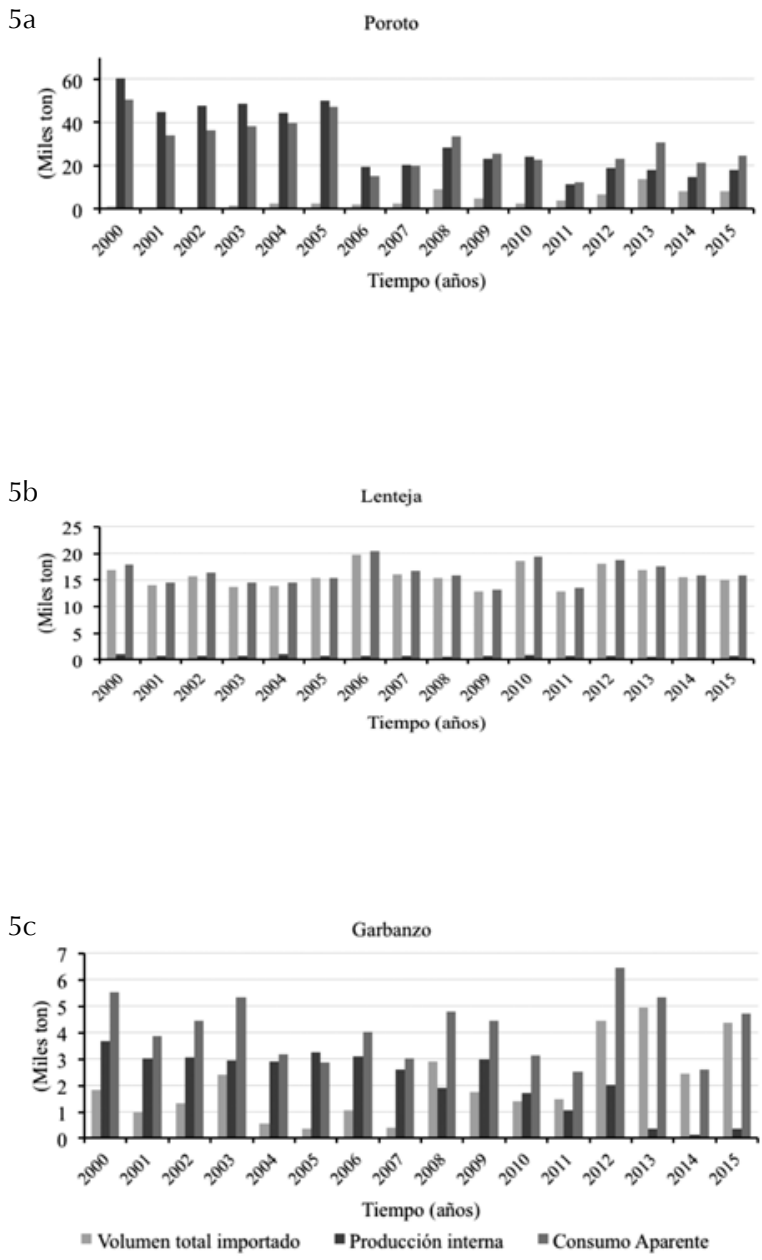

Figura 5. Volumen importado, Producción interna y consumo aparente de poroto, lenteja y garbanzo (total país, últimos 16 años). (Elaboración propia a partir de datos de ODEPA ${ }^{6}$.

Los acotados márgenes de ganancia indicados, están dados, además, por problemas entorno al manejo agronómico de las legumbres en el país.En este sentido, existe escaso desarrollo y transferencia tecnológica asociada a aspectos de manejo que influyen directamente en los rendimientos tales como: fertilización, requerimientos hídricos de las plantas en sus estados más críticos, uso adecuado de agroquímicos o de biofertilizantes, problemas de enfermedades, plagas y malezas, entre otros. Estos aspectos han generado sistemas agrícolas menos eficientes, con rendimientos bajos y con precios poco competitivos para el mercado.

\section{Beneficios a la salud del sistema agrícola}

En la actualidad, como pudo apreciarse en las estadísticas presentadas, el cultivo de legumbres en Chile evidencia un estado crítico desde un punto de vista económico, sin 
embargo, se debe rescatar de ellas los múltiples beneficios productivos y medioambientales que generan ${ }^{1-3}$ y que se describen a continuación:

1) Fijación de nitrógeno ( $N$ ) y aporte de $\mathrm{N}$ al suelo: En forma natural, cualquier planta toma $\mathrm{N}$ mineralizado desde el suelo, el que se encuentra en forma de iones nitrato $\left(\mathrm{NO}_{3}\right)$ o amonio $\left(\mathrm{NH}_{4}\right)$, y lo utilizan en su crecimiento. Sin embargo, las leguminosas (incluidas las legumbres), tienen además la capacidad de asociarse simbióticamente con bacterias altamente especializadas Ilamadas comúnmente rizobios (Rhizobium, Bradyrhizobium, Sinorhizobium, Mesorhizobium, entre otros géneros) y fijar $\mathrm{N}$ atmosférico; es decir, estas bacterias toman el $\mathrm{N}$ del aire y lo incorporan dentro de la planta a través de un proceso llamado fijación simbiótica de nitrógeno (FSN) ${ }^{9-12}$. En esta asociación, las bacterias realizan la FSN dentro estructuras llamadas nódulos (que contienen miles de bacterias), cuyo mecanismo y funcionamiento escontrolado tanto por la planta como por la bacteria ${ }^{10}$. Esta interacción rizobio-planta es específica, lo que significa que cada leguminosa es nodulada por su rizobio o rizobios característicos ${ }^{9}$.

Toda la actividad fijadora de $\mathrm{N}$ está organizada en función de una enzima, la nitrogenasa, presente en las bacterias y a través de la cual el nitrógeno atmosférico $\left(\mathrm{N}_{2}\right)$ se reduce a amoniaco $\left(\mathrm{NH}_{3}\right)$ el que se ioniza rápidamente a amonio $\left(\mathrm{NH}_{4}\right)$, siendo este último el que es incorporado a compuestos carbonados necesarios para la planta, entre ellos los aminoácidos. Para que este proceso ocurra, la bacteria requiere energía para su metabolismo, la que es aportada por la planta en forma de ATP a través de compuestos carbonados oxidables ${ }^{13}$. Cabe destacar que la eficiencia con que este mecanismo funciona, es decir, la cantidad de $\mathrm{N}$ fijado, dependerá de factores bióticos y abióticos asociados tanto a la planta leguminosa como a la bacteria en cuestión.

Así, por ejemplo, las condiciones ambientales a las que está sometida la planta, entre ellas: temperatura del aire y suelo, humedad y condiciones físicas del suelo (textura, estructura, profundidad, porosidad), pueden generar una mayor o menor fijación de $\mathrm{N}^{12,14}$. Por tanto, toda aquella condición de estrés a la cual pueda estar sometida la planta (falta de agua, excesos o bajas de temperaturas, falta de oxígeno en el suelo, etc.) afectará indirectamente a la bacteria y con ello la fijación de N. Asimismo, las aplicaciones excesivas de nitrógeno pueden inhibir el funcionamiento de la bacteria. Una forma práctica de evidenciarlo es revisar los nódulos formados en las raíces mediante su disección puesto que si presentan coloración rojiza (leghemoglobina en su interior), los nódulos estarán activos. En la Tabla 2, se presentan datos referenciales de $\mathrm{N}$ fijado por las legumbres ${ }^{11}$, pudiéndose observar que los valores pueden ser tan altos como $300 \mathrm{Kg}_{\text {de }} \mathrm{N} \mathrm{ha}^{-1} \mathrm{año}^{-1}$. Del total de $\mathrm{N}$ fijado, una parte queda retenida en los granos de las plantas para ser transformados a aminoácidos y proteínas u otros compuestos nitrogenados, pero otra parte permanece en tallos, raíces y nódulos, quedando disponible en el suelo una vez que la planta se coseche y/o cumpla su ciclo de vida (Tabla 3). En términos generales, se estima que por cada tonelada de materia seca producida por una especie de leguminosa se depositan en el suelo alrededor de $21 \mathrm{~kg}$ de $\mathrm{N}^{15}$. Por lo tanto, la FSN representa un aporte sustancial a los sistemas agrícolas, dado que el $\mathrm{N}$ resultante contribuye en la recuperación de las reservas naturales de nitrógeno orgánico en el suelo. Además, las bacterias que

Tabla 2

Rangos de nitrógeno atmosférico fijado por temporada

\begin{tabular}{|lc|}
\hline Cultivo de Legumbres & $\begin{array}{c}\text { N fijado } \\
\mathbf{k g ~ h a}^{-\mathbf{1}} \mathbf{~ a n ̃ o ~}^{-\mathbf{1}}\end{array}$ \\
\hline Haba & $100-300$ \\
Arveja & $50-270$ \\
Lupino & $40-300$ \\
Fréjol & $25-100$ \\
Adaptado de Urzúa ${ }^{8}$. & \\
\hline
\end{tabular}

Tabla 3

Contribución del nitrógeno fijado por legumbres, luego de la cosecha, al sistema suelo en distintos ambientes (valores promedio de dos temporadas)

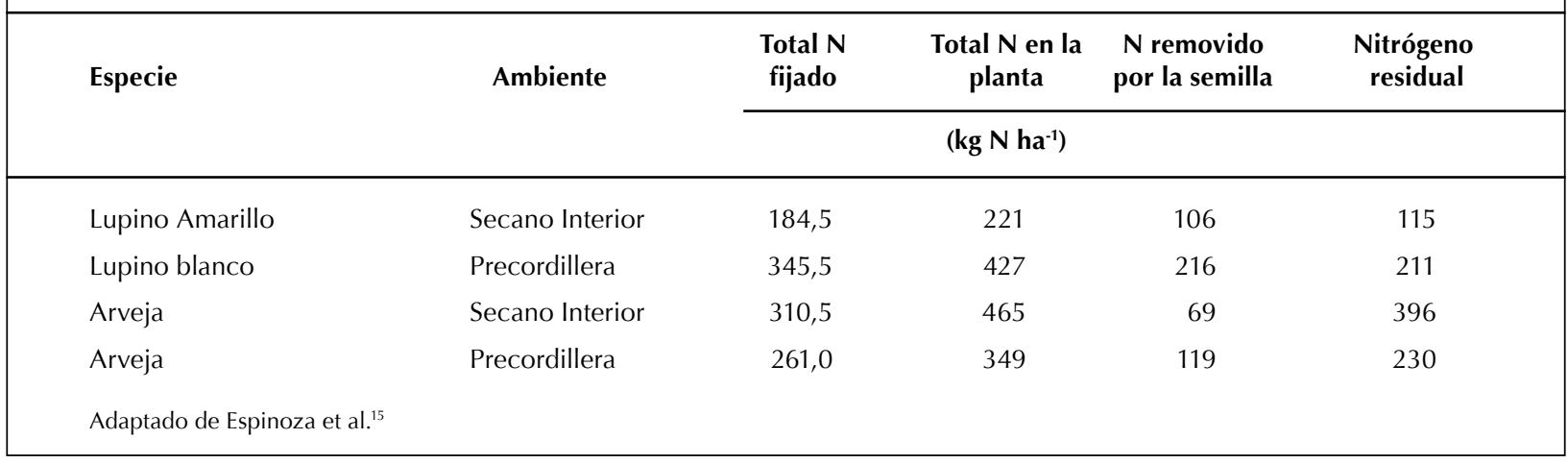


se asocian a las leguminosas son endobacterias (bacterias presentes dentro de las células de la raíz de la planta), por lo que el $\mathrm{N}$ es aportado directamente al interior de la planta, lo que implica que son nulas las pérdidas por lixiviación o volatilización de este $\mathrm{N}$. Este aspecto es trascendental si se compara con gran parte de los fertilizantes sintéticos que terminan siendo lixiviados o volatilizados, sobre todo cuando se ha hecho un uso irracional de ellos.

La disminución en el uso de fuentes sintéticas, permite que la emisión de gases de efecto invernadero (GEI) se reduzca por un menor uso y traslado de estos y, en consecuencia, en un ahorro sustancial de los costos en fertilización ${ }^{9}$. Todo ello indica que la fijación de $\mathrm{N}$ atmosférico es sustentable y no disruptiva de los procesos biológicos y ecológicos naturales que ocurren en el suelo ${ }^{16}$.

2) Aporte de materia orgánica al suelo: La incorporación de las leguminosas al suelo como abonos verdes, puede generar montos de $\mathrm{N}$ que varían entre los $70-300 \mathrm{~kg} \mathrm{ha}^{-1}$ año-1 para arveja, 80-380 para haba y $150-310 \mathrm{~kg} \mathrm{ha}^{-1} \mathrm{año}^{-1}$ en lupino, pudiendo este $\mathrm{N}$ ser utilizado por los cultivos que le suceden a las legumbres en la rotación ${ }^{11,17}$. Además del aporte de $\mathrm{N}$ y otros nutrientes, la materia orgánica que se incorpora al suelo contribuye al mejoramiento de sus propiedades físicas de este, al promover la formación de macroporos (de origen estructural), que permiten tanto una adecuada conductividad de agua y aire ${ }^{18,19}$, así como también una mayor retención de agua en el suelo ${ }^{20}$. Además, las leguminosas presentan raíces pivotantes, las que pueden actuar de forma directa sobre la estructura del suelo, uniendo y comprimiendo partículas, favoreciendo la estabilidad de los agregados, lo que, a su vez, aumenta el espacio poroso a lo largo del perfil de suelo y, con esto, mejoran los niveles de aireación, retención de agua e infiltración en la rizósfera ${ }^{21,22}$. Al finalizar el ciclo del cultivo, la descomposición de las raíces permite obtener materia orgánica en profundidad y los exudados radicales pueden estimular el crecimiento de hongos micorrízicos, es decir, organismos del suelo que viven simbióticamente con la mayoría de las raíces de las plantas, facilitándoles la toma de nutrientes de baja disponibilidad o de poca movilidad en el suelo, entre ellos el fósforo 9,17,22.

3) Disminución de problemas sanitarios (malezas, plagas y enfermedades): La incorporación de materia orgánica proveniente de las leguminosas, también puede tener efectos sobre la disminución de plagas o enfermedades presentes en el suelo. Estudios realizados con haba (Vicia faba L.) como cultivo de cobertura, demostraron que la presencia de Nacobbus aberrans y Globodera (nemátodos que dañan las raíces de las plantas) disminuyó entre un 30 y $42 \%$, luego que los residuos fueran incorporados ${ }^{23,24}$, atribuyendo este efecto a la baja relación $\mathrm{C} / \mathrm{N}$ del rastrojo de haba, una vez una vez que es incorporado al suelo. De igual manera, se ha estudiado el efecto nematicida de plantas de porotos usadas como mulch sobre Meloidogyne incognita en raíces de plantas de tomate, concluyendo que el efecto reductor de la población de este nemátodo fue similar al obtenido por el del producto comercial recomendado ${ }^{25}$. Además, incluir un cultivo de leguminosas (legumbres) en la rotación permite realizar un mejor control de malezas e interrumpir el ciclo de enfermedades que afectan a otros cultivos, disminuyendo así el uso de pesticidas ${ }^{26}$. Las leguminosas en rotación con cereales, a la vez que aportan nitrógeno y fósforo reducen la incidencia de malezas al retardar la aparición y aumento de biotipos de gramíneas como avenilla y ballica ${ }^{9}$, las cuales son resistentes a herbicidas selectivos en cultivos de la misma familia botánica (trigo, avena, cebada, triticale). Por otra parte, las leguminosas, por su cobertura y capacidad alelopática (compuesto químico liberado por una planta sobre el desarrollo y crecimiento de otra planta), evitan que las malezas proliferen, gracias a la secreción de compuestos fenólicos, tanto a nivel radical como producto de la descomposición del rastrojo ${ }^{25,27,28}$. Asimismo, ha sido ampliamente documentado el efecto sobre la incidencia de enfermedades en cereales de invierno. En este sentido, en estudios llevados a cabo en el sur de Chile, se observó que la inclusión de lenteja en la rotación con trigo, permitió aumentar por sobre un $50 \%$ el rendimiento de trigo, respecto al monocultivo (siembra de trigo después trigo). Los investigadores asociaron este efecto a la interrupción del hongo Gaeumannomyces graminis var. Tritici causante del "mal del pie" que afecto a las plantas de trigo en monocultivo y no así en rotación ${ }^{9}$. Adicionalmente, se ha observado que, para el caso de los cereales, contar con un pre-cultivo de leguminosas, puede influir no sólo en el rendimiento de los cereales sino también en la calidad del cereal ${ }^{29,30}$, registrándose aumentos entre el 1 y $8 \%$ en el contenido de proteína en el grano ${ }^{9}$.

4) Reducción de la huella hídrica: En general es más eficiente obtener calorías, proteínas y grasas a través de los productos vegetales que de los productos animales. En este sentido y analizando la huella hídrica de las legumbres respecto otras fuentes proteicas, Mekonnen y Hoskstra ${ }^{4}$ señalan que por cada gramo de proteína presente en la leche, huevos o pollo se requiere en promedio un $50 \%$ más de agua en comparación con la requerida por las legumbres, mientras que el volumen de agua usada asciende a 6 veces más cuando se compara con carne bovina. De igual manera, los autores estudiaron las dietas con y sin carne, pero con montos equivalente de proteínas en base a legumbres y frutos secos, resultando en una reducción promedio de un $30 \%$ en la huella hídrica de la dieta vegetariana respecto de la dieta con carne.

Para lograr que las legumbres sean eficientes en el uso del agua, se requiere hacer un buen manejo del riego, sobre todo porque en Chile el clima preponderante de la zona productiva es el clima Mediterráneo (precipitaciones se presentan principalmente en los meses de invierno y luego hay sequía terminal) y, además, porque las precipitaciones han ido disminuyendo en la zona productora de legumbres, como consecuencia de los efectos del cambio climático ${ }^{31}$. Por tanto, se requiere de riegos eficientes, es decir, que se prioricen en las etapas críticas del cultivo (inicio de 
floración hasta llenado de granos) que son las que más inciden en el rendimiento de las legumbres ${ }^{32}$. Frente a este mismo escenario y específicamente en poroto, la gran diversidad genética existente en Chile $^{33}$ permite hacer frente a las restricciones hídricas utilizando variedades de ciclos más $\operatorname{cortos}^{34}$. En este sentido, el principal mecanismo de adaptación de las legumbres, para un alto rendimiento, es a través del escape a la sequía terminal, mediante fechas de siembra tempranas que permitan una rápida cobertura del suelo y, consecuentemente, una floración y llenado de granos antes del inicio de la sequía. Esto permitirá una mayor partición de materia seca hacia la producción de granos, resultando en un mayor índice de cosecha y una mayor eficiencia en el uso del agua (EUA). En arveja, por ejemplo, se han determinado valores de EUA que varían entre 6 y $15,3 \mathrm{~kg}$ de granos $/ \mathrm{ha} / \mathrm{mm}$ de agua evapotranspirada, mientras que en haba estos valores fluctúan entre 7,7 y 11,4 y en garbanzo entre 2,6 y $7,7 \mathrm{~kg}$ de granos $/ \mathrm{ha} / \mathrm{mm}$ de agua evapotranspirada ${ }^{34}$.

\section{Problemáticas de manejo de las legumbres en Chile}

Son varios los problemas de manejo que enfrentan hoy las legumbres en Chile, sobre todo y tal como se ha indicado anteriormente, el rubro está principalmente en manos de pequeños agricultores que no cuentan con acceso a nuevas tecnologías. Por tanto, uno de los principales problemas está relacionado con la mecanización de las labores agrícolas como la siembra y cosecha. Existen actualmente en Chile siembras de cultivos de legumbres que aún se realizan a mano, este es el caso del haba, que debido al gran tamaño de sus semillas, imposibilita utilizar máquinas de precisión y muchos agricultores terminan sembrando manualmente ${ }^{35}$.

En otras especies como poroto y arveja, las siembras se realizan con máquinas de tarros o cerealeras, no haciendo regulaciones adecuadas para ninguno de los dos tipos de semillas. Este hecho genera graves problemas en el establecimiento del cultivo dado que la distribución de las plantas resulta ser muy desuniforme y por ende crecen con diferentes niveles de competencia entre ellas, generando problemas de manejo como lo son presencia de malezas (en los espacios donde hay menos plantas), riegos no óptimos (las malezas consumen gran parte del agua y/o hay pérdida del agua por evaporación directa desde el suelo) y finalmente problemas en la cosecha por desigualdad en la madurez de los granos.

Asociado a la mecanización y como parte del problema, está el tipo de variedades que se siembran en el país, puesto que la mayor parte de ellas, son de hábitos de crecimiento indeterminado, lo que significa que al momento de la cosecha terminan tendiéndose en el suelo, imposibilitándose la cosecha mecanizada. Por ello, algunos productores, arrancan primero las plantas manualmente y posteriormente las trillan con máquinas estacionarias habilitadas para estos y otros cultivos. Otro de los problemas tiene relación con el tipo de semillas utilizadas para las siembras, dado que en general son de baja calidad debido a que los agricultores usan su propia semilla o la adquieren en empresas que no cumplen las condiciones mínimas de rigurosidad, en términos de manejo de un semillero.

Cabe destacar que, en el rubro de las legumbres, no existe el uso de semilla certificada, es decir aquella que cuenta con un porcentaje de germinación, pureza varietal y física asegurada. Este hecho está asociado al alto costo que significa la compra de este tipo de semillas para los agricultores. Por tanto, en el caso de los porotos, con frecuencia vienen semillas mezcladas, es decir, coexisten cultivares distintos en un mismo lote, lo que atenta directamente con un correcto establecimiento y desarrollo del cultivo ${ }^{36}$. En otros casos, la variedad no corresponde a la comprada o la semilla viene con daños físicos en su testa, provocado por un manejo inadecuado durante el traslado de la semilla y su almacenamiento, disminuyendo su capacidad de generar plántulas normales y homogéneas ${ }^{37}$.

El uso de semilla de no certificada conlleva a problemas sanitarios, dado que un alto porcentaje de los agentes causantes de enfermedades en las plantas de legumbres pueden provenir de la semilla y, por lo tanto, éstas pueden ser un medio de propagación de esas enfermedades ${ }^{3}$, más aún cuando, en general, los productores no aplican fungicidas a sus semillas. Los problemas sanitarios, por tanto, son aspectos de manejo de importancia en este grupo de cultivos y sobre todo aquellos que tienen relación con enfermedades de difícil control, como son aquellas transmitidas por las semillas entre ellas los virus, siendo estos últimos los más graves dado que actualmente no cuentan con control químico. En el caso específico de poroto, el virus del mosaico común (VMCF) y el mosaico amarillo (VMAF) han sido los de mayor importancia generando pérdidas de rendimiento que superan el $50 \%$ y alterando la calidad del producto cosechado ${ }^{38}$.

En lenteja, uno de los problemas sanitarios de importancia han sido las royas (Uromyces fabae Pers.), siendo esta enfermedad una de las principales responsables de la disminución de la superficie sembrada de este cultivo en Chile debido a las altas pérdidas de rendimiento que genera $^{39}$. En garbanzo, las enfermedades radicales han sido las de mayor importancia, siendo la causada por Fusarium solani la más grave ya que causa marchitez parcial o total de las plantas ${ }^{40}$; al ser una enfermedad presente en el suelo, es de difícil control, salvo que se realce rotación de cultivos, manejo que no siempre se lleva a cabo $^{41}$.

\section{Desafíos más importantes}

A pesar de que existe más conciencia de la importancia de las legumbres en la salud y en la prevención de algunas enfermedades no transmisibles, aún no se ha tomado cabal conciencia de los beneficios que generan en el entorno donde se cultivan y menos aún se ha avanzado en la adopción de nuevas tecnologías de producción. Por ello, a continuación se dan a conocer los desafíos más importante en torno a este último tema. 
Hacia la mecanización: Esta es una condición necesaria para la modernización de la agricultura y a través de la cual se logra aumentar la eficiencia y la productividad de las empresas agrícolas. La posibilidad de mecanizar el cultivo de las legumbres se centra en:

(i) Aspectos genéticos, a través de la generación de variedades decrecimiento determinado, evitando con ello la tendedura y homogeneizando la cosecha.

(ii) Aspectos de manejo agronómico, como densidades de siembra o control demalezas adecuado, que eviten el crecimiento en altura delas plantas de legumbres por competencia por luz y, por ende, desfavorezcan la tendedura.

(iii) Se requiere innovar el uso de sistemas de labranza vertical (arados tipo cincel o subsoladores) que permitan mejorar la capacidad de infiltración de agua en los suelos, aumentando su disponibilidad sobre todo en zonas donde las precipitaciones son escasas.

Utilización de semillas de mejor calidad: Uno de los requisitos fundamentales para la producción de legumbres es lograr un buen establecimiento de las plantas en el terreno. Para ello se debe contar con semilla de calidad, es decir, que presente un alto porcentaje de germinación y que esté libre de enfermedades o plagas que puedan dañar a las plantas durante el establecimiento y/o crecimiento ${ }^{42}$. La utilización de semilla certificada, por tanto, podría constituirse en un gran desafío para la producción de legumbres en Chile, dado que actualmente la mayor parte de las siembras se llevan a cabo con semillas producidas por los propios productores, sin contar con la rigurosidad requerida para la producción de semilla certificada, la que debe contar con: alto porcentaje de germinación, pureza varietal, pureza física y pureza sanitaria ${ }^{42,43}$. En la transición hacia el uso de semilla certificada, y considerando que un porcentaje alto de agricultores produce su propia semilla, se podrían generar instancias de capacitación para los productores en torno al manejo requerido para optimizar la producción de semillas, entre ellos: eliminación de plantas enfermas, principalmente de aquellas que se trasmiten por las semillas; evitar daños mecánicos durante la trilla de los granos puesto que se afecta finalmente su capacidad germinativa; limpiar y seleccionar la semilla; contar con condiciones óptimas de almacenaje, evitando con ello que la semilla se envejezca y deteriore.

Mejoramiento genético: Uno de los aspectos claves en los programas de mejoramiento genético es contar con una amplia diversidad genética $y$, en ese contexto, Chile tiene grandes ventajas, puesto que en el país existe un Sub-Centro de diversidad genética para poroto ${ }^{44}$. Por ello, se podría avanzar rápidamente en los programas de mejoramiento genético pues se cuenta con el material necesario para ello. Sin embargo, a nivel nacional no existe ningún programa de mejoramiento genético vigente para esta especie ni para el resto de las legumbres. Si estos programas se activarán, los principales objetivos del mejoramiento genético deberían ser producción y calidad de los granos.

Desde el punto de vista de la producción, los desafíos más importantes son:

(i) Resistencia a enfermedades: Entre los problemas sanitarios de importancia y en los que hay que generar plantas resistentes y/o tolerantes, se encuentran los virus, muchos de los cuales son trasmitidos a través de las semillas. No obstante, un desafío no menor, es la promoción de controles preventivos (ej., eliminar insectos que puedan diseminar la enfermedad en el campo) y el estudio de la biología de las enfermedades y los factores que inciden en su interacción con el medio, a fin de establecer con propiedad mecanismos que mejoren la tolerancia o resistencia de las plantas ${ }^{45}$.

(ii) Nodulación y fijación de N: Existen especies de legumbres que presentan escasa capacidad de nodulación bajo condiciones adversas, por lo que la ventaja de incorporar $\mathrm{N}$ al sistema se pierde ${ }^{46}$. El desafío, por tanto, es buscar cepas de rizobios, principalmente nativas, que sean capaces de asociarse simbióticamente con las legumbres de manera eficiente, es decir, que logren aportar los requerimientos de $\mathrm{N}$ de las plantas para un óptimo rendimiento, sobre todo bajo condiciones adversas. Para ello, el conocimiento de la biodiversidad de estos microrganismos es clave. En este sentido, Baginsky et al. ${ }^{12}$, señalan que en Chile existe una amplia diversidad de especies de Rhizobium que se asocian con poroto, siendo $R$. leguminosarum la especie que presenta la mayor diversidad ${ }^{7}$. Asociado a este mismo tema, y como un desafío relevante para el país, está la necesidad de desarrollar empresas que vendan inoculantes fabricados con cepas nativas adaptadas a las diferentes condiciones nacionales, de tal manera que los agricultores cuenten con una herramienta de fertilización biológica para sus campos ${ }^{47}$. Unido a ello, se requiere promover este conocimiento a través de días de campo donde se den a conocer las ventajas de este sistema a los agricultores.

(iii) Características agronómicas específicas: Se requiere (a) variedades aptas para ser cosechadas con máquina y mejorar la eficiencia de esta labor, reduciéndose con ello el costo de la mano de obra cada vez más escasa. Para ello, los desafíos principales son plantas de hábitos de crecimiento determinado (algo se ha avanzado en porotos para consumo hortícola); en este caso, se busca principalmente plantas pequeñas y de maduración homogénea; plantas erectas y con el primer nudo con vainasa suficiente altura del suelo $(20 \mathrm{~cm}$ aprox.); (b) cultivares que presenten un alto índice de cosecha, es decir, alta proporción de granos respecto de la biomasa total de la planta, de tal manera que se genere mayor rendimiento con menor acumulación de biomasa. Un ejemplo de ello, son variedades que producen más vainas por nudo o más granos por vaina. De esta manera, se logra una mayor eficiencia en el 
uso de los recursos disponibles.

(iv) Adaptación al medio: Frente al inminente cambio climático ${ }^{48,49}$, es necesario contar con variedades que se adapten a este nuevo escenario y que toleren diferentes tipos de estrés, entre ellos: sequías e inundaciones, priorizando variedades de ciclos cortos; altas o bajas temperaturas (heladas); respuesta al fotoperíodo. Además, el mejoramiento genético deberá buscar variedades que se adapten a suelos salinos, donde además hay escasez hídrica; a suelos de escasa fertilidad, debido a que las legumbres han sido desplazadas por cultivos más rentables, hacia zonas más marginales.

Desde el punto de vista de la calidad alimenticia de los granos, los desafíos más importantes son:

(i) Aumento en el contenido de proteínas y mejoras en el perfil aminoacídico, sobretodo aquellos aminoácidos limitantes como los azufrados. Estudios han determinado que, en especies como la arveja, mientras más grande la semilla, mayor contenido de proteína ${ }^{50}$;

(ii) Aumento en el contenido y disponibilidad de minerales, vitaminas, fibras y antioxidantes. Entre los minerales más importantes está el calcio, hierro y zinc y entre las vitaminas se encuentra la tiamina, riboflavina, niacina, vitamina B6 y ácido fólico. En cuanto a los compuestos antioxidantes sobre salen los fitoesteroles ${ }^{51}$;

(iii) Eliminación de compuestos tóxicos como las hematoaglutininas presentes en lentejas, habas y porotos; lectinas en porotos; factores productores de flatulencia en lenteja y porotos, entre otros;

(iv) Facilidad de cocción. Este es un punto clave en lo que respecta a aspectos culinarios, buscando aquellas variedades que presenten testas más delgadas y que permitan la entrada de agua para su ablandamiento. Así, por ejemplo, en lupino Lupinus albus la cubierta seminal puede llegar a corresponder hasta a un 15\% respecto del peso del grano, siendo esta difícil de digerir y de bajo aporte nutricional ${ }^{52}$.

Políticas Públicas: Con el objeto de mejorar la situación actual de las legumbres en el país, se requiere una mayor participación del Estado en la generación de políticas públicas. Actualmente, la Ley $\mathrm{N}^{\circ} 20.142$ que decreta la entrega de "Incentivos para la Sustentabilidad Agroambiental de los Suelos Agropecuarios" o programa SIRSD-S53, tiene como principal objetivo recuperar el potencial productivo de los suelos a través de una bonificación estatal entregada mediante concurso público. Esta Ley considera el cultivo de las leguminosas como ente principal en: el establecimiento de praderas o para la recuperación de praderas degradadas; la incorporación de abono verde; rotaciones de cultivo, siendo obligatorio el establecimiento de una legumbre como cultivo de cabecera y como opción de asociación a un cereal el segundo año; uso para el control de cárcavas ${ }^{53}$. A nivel internacional existen políticas públicas como la Política Agraria Comunitaria (PAC 2014-2020) implementada por la Unión Europea (UE) la cual incentiva a los agricultores a "incrementar su producción y productividad y a diversificar sus cultivos sin dejar de lado la protección del medio ambiente y la adaptación al cambio climático", otorgándoseles un Pago Verde o "Greening", a los productores. Este hecho incentivó el uso de legumbres en el UE, provocando el aumento de la superficie de cultivo y reactivación de su comercio ${ }^{54}$.

\section{CONSIDERACIONES FINALES}

El escenario en el cual están actualmente insertas las legumbres no es muy auspicioso, dado principalmente por agricultores con escasa tecnología, rendimientos bajos y que se han mantenido por más de 40 años, siembras cada vez más escasas e importaciones que han ido en aumento a través del tiempo, a precios muy poco competitivos para los productores. Pese a ello, y dadas las ventajas antes descritas y con el afán de rescatar cultivos, como el poroto, que están estrechamente ligados a la identidad nacional, se requiere un input de innovación tanto tecnológica como gastronómica a este grupo de cultivos tan alimenticios y tan necesarios desde un punto de vista agronómico.

Ante esto, la potenciación de las legumbres en el quehacer agropecuario requiere del esfuerzo conjunto del sector público, investigadores, profesionales, agricultores, consumidores, chefs, entre otros, es decir, enlazar la cadena productiva de forma tal de hacerla más eficiente y capaz de satisfacer las necesidades de cada sector. El escenario climático actual plantea la necesidad de generar alternativas que permitan compensar la demanda de alimentos en el futuro. En torno a la producción de legumbres, es preciso identificar cultivares que tengan una mayor eficiencia en el uso del agua o ciclos más cortos, que les permitan escapar del período de mayor demanda hídrica; además, seleccionar aquellos con resistencia a enfermedades y a altas temperaturas. Todos estos factores deben ser abordados desde distintas disciplinas, en particular, la fisiología vegetal, la agronomía, el mejoramiento genético, la simulación de modelos de cultivos, sistemas de información geográfica y el ordenamiento territorial ${ }^{19,49}$. La demanda tecnológica, para el cultivo de legumbres en Chile, evidencia la necesidad de realizar mejoras a nivel de los pequeños agricultores en los sistemas de labranza, fertilización, siembra y cosecha. Se requiere enfatizar en la capacidad de las legumbres de fijar nitrógeno y en la necesidad de generar variedades aptas para la cosecha mecanizada.

Finalmente, técnicos, profesionales agrícolas y agricultores están llamados a mejorar los sistemas de manejo actuales que permitan una mejor utilización del recurso hídrico y suelos, requiriendo para esto capacitación constante a fin de actualizar sus conocimientos y adquirir nuevas herramientas que permitan satisfacer la demanda tecnológica de los sistemas y entregar un mejor servicio a pequeños agricultores. Es necesario alentar las conexiones de la cadena para una mayor producción de estos cultivos, teniendo en cuenta que, para el año 2050, se estima que 
existirán nueve mil millones de habitantes en este mundo, la mayor parte en países subdesarrollados, sin carne, casi sin pan, con tierras poco o nada fértiles, con la necesidad añadida de mantener un ambiente limpio sin contaminante, por tanto, vale la pena, revitalizar este alimento y cultivo.

\section{BIBLIOGRAFÍA}

1. Strong W, Harbison J, Nielsen R, Hall B and Best E. Nitrogen availability in a Darling Downs soil following cereal, oilseed and grain legume crops. 2. Effects of residual soil nitrogen and fertiliser nitrogen on subsequent wheat crops. Aust J of Exp Agric 1986; 26: 353-359.

2. Jensen E, Peoples M and Hauggaard-Nielsen H. Faba bean in cropping systems. Field Crops Res 2010; 115: 203-216.

3. Köpke $U$ and Nemecek T. Ecological services of faba bean. Field Crops Res 2010; 115: 217-233.

4. Mekonnen M and Hoekstra A. A global assessment of the water footprint of farm animal products. Ecosystems 2012; 15: 401-415.

5. ODEPA (Agricultural Office of Studies and Policies). Annual series by product of exports and imports [Internet Database]. Chile: ODEPA; 2017. Available on: http://www.odepa.cl/ series-anuales-por-producto-de-exportaciones-importaciones/

6. ODEPA (Agricultural Office of Studies and Policies). Annual national information of planted surface, production and yields. Chile: ODEPA; 2017. Available on: http://www.odepa.cl/wpcontent/uploads/2017/04/CultivosAnualesHistorico-03.04. 2017.x/s

7. ODEPA (Agricultural Office of Studies and Policies). Annual regional information of planted surface, production and yields. [Internet Database]. Chile: ODEPA; 2017- Available on: http:// www.odepa.cl/wp-content/uploads/2017/02/cultivosregional_ 03.04.2017.x/s

8. Figueroa $V$, Silva P, Baginsky C, Pinheiro A, Pizarro $T$ and Rodríguez $L$. The pulses queen. Beans: from our origins to the table. Indualimentos 2014; 86: 90-97.

9. Mera M and Rouanet IL. Contribution of the grain legume on rotation with cereals: a review. In: Acevedo $E$, editor. Sustentability in annual crops. Chile: University of Chile 2003; p. 135-156.

10. Mayz-Figueroa J. Nitrogen biological fixation. Rev UDO Agr 2004; 4(1): 1-20.

11. Urzúa H. Benefit of nitrogen biological fixation in Chile. Sci Invest 2005; 32 (2): 133-150.

12. Baginsky C, Brito B, Scherson R, Pertuzé $R$, Seguel O, Cañete A et al. Genetic diversity of Rhizobium from nodulating beans grown in variety of Mediterranean climate soils of Chile. Arch Microbiol 2015; 197: 419-429.

13. Mayz J. Symbiosis Legume/Rhizobia. Venezuela: Ed. Inst. Invest. Agrop. IIAPUDO, East University; 1997. p. 113.

14. Berg G and Smalla K. Plant species and soil type cooperatively shape the structure and function of microbial communities in the rhizosphere. FEMS Microbiol Ecology 2009; 68: 1-13.

15. Espinoza S, Ovalle C, Zagal E, Matus I, Tay J, Peoples MB and del Pozo A. Contribution of legume to wheat productivity in Mediterranean environments of Central Chile. Field Crops Res 2012; 133: 150-159.

16. Bender SF, Wagg C and van der Heijden MGA. An underground revolution: biodiversity and soil ecological engineering for agricultural sustainability. Trends Ecol Evol 2016; 1-13.

17. Cerrato $R$ and Alarcón A. Soil microbiology on the sustainable agriculture. Sci Ergo Sum 2001; 8: 175-185.
18. Kempler Kemper $W$ and Rosenau R. Aggregate estability and size distribution. In: Klute A, Editor. Methods of soil analysis. Vol. 1. Physical Methods. 2nd ed. Madison, Wisconsin, USA; ASA and SSSA; 1986. p. 425-442.

19. Brady Brady NC and Weil RR. Elements of the nature and properties of soils. Upper Saddle River, New Jersey, USA: Prentice Hall; 2000.

20. Hudson Hudson B. Soil organic matter and available water capacity. J Soil Water Conserv 1994; 49: 189-194.

21. Sultani M, Gill M, Anwar M and Athar M. Evaluation of soil physical properties as influenced by various Green manuring legumes and phosphorus fertilization under rain fed conditions. Int J Environm Sci Tech 2007; 4(1): 109-118.

22. FAO (Food and Agriculture Organization of the United Nations). Soils and Pulses. Symbiosis for life. Rome: FAO 2016; p. 114.

23. Iriarte L, Franco J and Ortuño N. Effect of organic mature on the nematodes population and potato production. Rev Latinoam de la papa 1998; 11: 149-163.

24. Pacajes G, Franco J, Estrella R and Main G. Effect of different crops and agricultural practices on the multiplication of Potato Cyst Nematode (Globodera spp.) in Bolivia. Rev Latinoam de la papa 2002; 13:52-65.

25. Caamal-Maldonado JA, Jiménez-Osornio JJ, Torres-Barragán $A$ and Anaya AL. The use of allelopathic legume cover and mulch species for weed control in cropping systems. Agron J 2001; 93: 27-36.

26. Nemecek T, von Richthofenb J, Duboisc G, Castad P, Charlese $R$ and Pahlf $H$. Environmental impacts of introducing grain legumes into European crop rotations. Eur J Agron 2008; 28: 380-393.

27. Ortiz-Ceballos A, Aguirre J, Salgado S and Ortiz-Ceballos G. Maize-velvet bean rotation in summer and winter milpas: a greener technology. Agronomy Journal 2015; 107: 330-336.

28. Liebman M and Ohno T. Crop rotation and legume residue effects on weed emergence and growth: applications for weed management. In: Hatfield $J L$, Buhler DD and Stewart $B A$, editors. Integrated weed and soil management. Michigan, USA: Ann Arbor Press 1998; p. 181-222.

29. López-Bellido L, Fuentes M, Castillo J and López-Garrido F. Effects of tillage, crop rotation and nitrogen fertilization on wheat-grain quality grown under rainfed Mediterranean conditions. Field Crops Res 1998; 57: 265-276.

30. Galantini J, Landriscini M, Iglesias J, Miglierina A and Rosell $R$. The effects of crop rotation and fertilization on wheat productivity in the Pampean semiarid region of Argentina: 2. Nutrient balance, yield and grain quality. Soil Tillage Res 2000; 53(2): 137-144.

31. Garreaud R. Climate change: physics bases and impacts in Chile. Rev Tierra Adentro 2011; 93.

32. Mwanamwenge J, Loss SP, Siddique KHM and Cocks PS. Effect of water stress during floral initiation, flowering and podding on the growth and yield of faba bean (Vicia faba L.). Euro J of Agron 1999; 11: 1-11.

33. INIA (Agricultural Investigation Institute) [Web site]. Chile: INIA; [access 03 may 2017]. Plant breeding banks [app. 3 screens]. Available on: http://www.inia.cl/red-de-bancosde-germoplasma/bancos-fitogenetico/

34. Siddique KHM, Regan KL, Tennant D and Thomson BD. Water use and water use efficiency of cool season grain legumes in low rainfall Mediterranean-type environments. Eur J of Agron 2001; 15: 267-280.

35. Ansoleaga A, Salinas M and Cartes and LeBert Co. Results 
and lessons on faba bean baby production. Chile: Ograma Ltda 2010; p. 34.

36. Faiguenbaum H. Tillage, sowing and production of the principal crops of Chile. Santiago, Chile: Vivaldi y Asociados 2003; p. 760.

37. Romano A, Argüello J, Teves I, De Pascuale N, Oddone G, Cazón L. Mechanic damage in seed of two bean cultivar (Phaseolus vulgaris L.), impact on germination physiology. Idesia 2014; 32: 17-15.

38. Sepúlveda P. Increase the viruses of bean in Chile. Tierra Adentro 1995; 1: 30-33

39. Peñaloza E, Tay J and France A. Calpún-INIA, Lentil crop of a large seed size and rust-resistant. (Lens culinaris L.). Agric Téc 2007; 67(1): 68-71.

40. France, A. Chickpea disease in clay soil. In: Manual of chickpea production. Recommendation for a clay soil. Boletín INIA 2006; 143: 67-82.

41. Baginsky, C. Chickpea: A crop of soon recovery in Chile. In: Legume Perspectives. The Timeless Chickpea. J Int Legume Soc 2014; 3: 39.

42. Graham $P$ and Vance $C$. Legumes: importance and constraints to greater use. Plant Physiol 2003; 131: 872-877.

43. Tay J. Why prefer certificated seed on the use of improvement varieties?, Inf Agr Bioleche-INIA Quilamapu 1999; 17-19.

44. Bascur $G$ and Tay J. Collection, characterization and use of genetic variation in Chilean bean germplasm (Phaseolus vulgaris L.). Agric Téc 2015; 65: 135-146.

45. Rubiales D, Fondevilla S, Chen W, Gentzbittel L, Higgins $T$, Castillejo $M$ et al. Achievements and challenges in legume breeding for pest and disease resistance. Crit Rev Plant Sci 2015; 34: 195-236.

46. Duc G, Agrama H, Bao S, Berger J, Bourion V, De Ron AM, Gowda CLL, Mikic A, Millot D, Singh KB, Tullu A,
Vanderberg A, Vaz Patto MC, Warkentin TD and Zong X. Breeding annual grain legumes for sustainable agriculture: new methods to approach complex traits and target new cultivar ideotypes. Crit Rev Plant Sci 2015; 34: 381-411.

47. Dreaker $R$, Rughley $R$ and Kennedy I. Legume seed inoculation technology. A review, Soil Biol and Biochem. 2004; 36(2004): 1275-1288.

48. Intergovernmental Panel on Climate Change (IPCC). Climate change 2013. The Physical science basis, Summary for policymakers, technical summary and frequently asked questions. Working group I, contribution to the fifth Assessment report of the OPCC; 2013.

49. Vadez V, Berger J, Warkentin T, Asseng S, Ratnakumar $P$, Chandra KP et al. Adaptation of grain legumes to climate change: A review, Agron Sustain 2012; 2012: 31-44.

50. Cubero /J and Moreno MT. Pulses. Madrid, Spain: MundiPrensa 1983; p. 359.

51. Ryan E, Galvin K, O'Connor TP, Maguire $A R$ and $O^{\prime}$ Brien NM. Phytosterol, tocopherol and squalene content and fatty acid profile of selected seeds, grains, and legumes. Plant Foods for Human Nutr 2007; 62: 85-91.

52. Jansen P. Lupinus albus [on Internet]. Record from Protabase. Brink M. Bclay G. (eds) PROTA (Plant Resources of Tropical Africa / Ressources végétales de l'Afrique tropicale). Wageningen, Netherlands. 2006. Available on: http:// www.fao.org/docs/eims/upload/206604/7_1_4_proposals. pdf

53. System of Incentives for the Agro-Environmental Sustainability of Agricultural Soils. Law N²0412, decret $N^{\circ} 4$ of 6 march 2017.

54. Torres A, Rubio J, Millán T, Eraso E and Solís I. The role of the legume on the PAC: reality and perspectives. Rev Ambienta 2015; 112. 